\title{
Method for 3D Rendering Based on Intersection Image Display Which Allows Representation of Internal Structure of 3D objects
}

\author{
Kohei Arai ${ }^{1}$ \\ 1Graduate School of Science and Engineering \\ Saga University \\ Saga City, Japan
}

\begin{abstract}
Method for 3D rendering based on intersection image display which allows representation of internal structure is proposed. The proposed method is essentially different from the conventional volume rendering based on solid model which allows representation of just surface of the 3D objects. By using afterimage, internal structure can be displayed through exchanging the intersection images with internal structure for the proposed method. Through experiments with CT scan images, the proposed method is validated. Also one of other applicable areas of the proposed for design of 3D pattern of Large Scale Integrated Circuit: LSI is introduced. Layered patterns of LSI can be displayed and switched by using human eyes only. It is confirmed that the time required for displaying layer pattern and switching the pattern to the other layer by using human eyes only is much faster than that using hands and fingers.
\end{abstract}

Keywords-3D object display; volume rendering; solid model; afterimage; line of sight vector

\section{INTRODUCTION}

Computer input by human eyes only is proposed and implemented [1]-[3] together with its application to many fields, communication aids, electric wheel chair controls, having meal aids, information collection aids (phoning, search engine, watching TV, listening to radio, e-book/e-comic/elearning/,etc., domestic helper robotics and so on [4]-[15]. In particular, the proposed computer input system by human eyes only does work like keyboard as well as mouse. Therefore, not only key-in operations but also mouse operations (right and left button click, drag and drop, single and double click) are available for the proposed system.

It is well known that hands, fingers operation is much slower than line of sight vector movements. It is also known that accidental blink is done within 0.3 second. Therefore, the proposed computer input system by human eyes only decides the specified key or location when the line of vector is fixed at the certain position of computer display for more than 0.3 second. In other words, the system can update the key or the location every 0.3 second. It is fast enough for most of all application fields.

Meanwhile, 3D image display and manipulation can be done with 3D display. Attempts are also done with 2D display for 3D image display and manipulation, on the other hands. Most of previous attempts are based on touch panel based manipulation by hands and fingers. As aforementioned, eyes operations are much faster than hands \& fingers operations. Therefore, 3D image display and manipulation method by human eyes only is proposed in this paper.

The proposed method is essentially different from the conventional volume rendering based on solid model which allows representation of just surface of the 3D objects. By using afterimage, internal structure can be displayed through exchanging the intersection images with internal structure for the proposed method. Through experiments with CT scan images, the proposed method is validated. Also one of other applicable areas of the proposed for design of 3D pattern of Large Scale Integrated Circuit: LSI is introduced. Layered patterns of LSI can be displayed and switched by using human eyes only. It is confirmed that the time required for displaying layer pattern and switching the pattern to the other layer by using human eyes only is much faster than that using hands and fingers.

The following section describes the proposed method for $3 \mathrm{D}$ volume rendering together with theoretical background followed by some experiments with CT scan of imagery data. Then its applicability for LSI pattern design of 3D display of the layered pattern is discussed. After that, conclusion is described together with some discussions.

\section{PROPOSED METHOD AND SYSTEM}

\section{A. Basic Idea for the Proposed Method}

$3 \mathrm{D}$ model that displays a picture or item in a form that appears to be physically present with a designated structure. Essentially, it allows items that appeared flat to the human eye to be display in a form that allows for various dimensions to be represented. These dimensions include width, depth, and height.

3D model can be displayed as a two-dimensional image through a process called $3 D$ rendering or used in a computer simulation of physical phenomena. The model can also be physically created using 3D printing devices.

Models may be created automatically or manually. The manual modeling process of preparing geometric data for 3D computer graphics is similar to plastic arts such as sculpting. 
There are some previously proposed methods for 3D display such as tracing object contour and reconstruct 3D object with line drawings, and wireframe representation and display 3D object with volume rendering. There is another method, so called OCT: Optical Coherence Tomography. It, however, quit expensive than the others.

Fundamental idea of the proposed method is afterimage. Response time of human eyes is that the time resolution of human eyes: $50 \mathrm{~ms} \sim 100 \mathrm{~ms}$. An afterimage or ghost image or image burn-in is an optical illusion that refers to an image continuing to appear in one's vision after the exposure to the original image has ceased. One of the most common afterimages is the bright glow that seems to float before one's eyes after looking into a light source for a few seconds.

Afterimages come in two forms, negative (inverted) and positive (retaining original color). The process behind positive afterimages is unknown, though thought to be related to neural adaptation. On the other hand, negative afterimages are a retinal phenomenon and are well understood.

Example of 3D image on to 2D display is shown in Figure 1. This is the proposed system concept. In the example, "A" marked 3D image of multi-fidus which is acquired with $\mathrm{CT}$ scanner is displayed onto computer screen. "B", "C", ... are behind it.

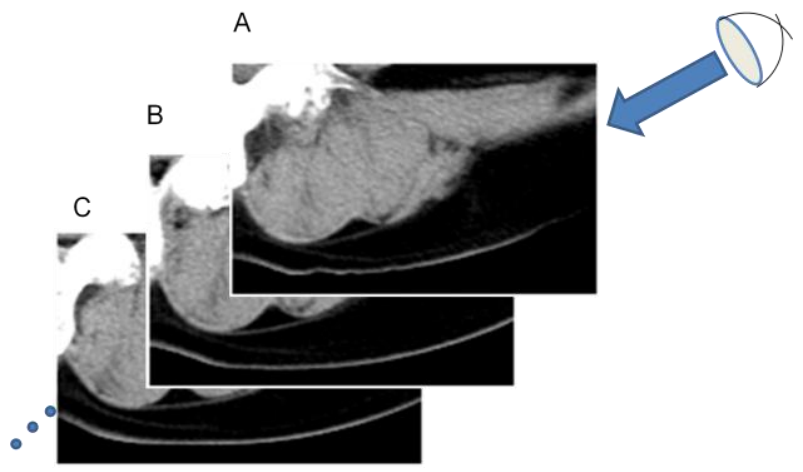

Fig. 1. System Concept

Such this layered 3D images are aligned with depth direction. Attached character "A" to "Z" are transparent and displayed at just beside the layered image at the different locations.

Therefore, user can recognize the character and can select one of those characters by their eye. Arrow shows the line of sight vector. Curser can be controlled by human eyes only. By sweeping the character, 3D images are displayed by layer by layer. Therefore, it looks like time division delay of 3D images.

\section{B. Automatic Mode and Eye Cotroll Mode}

There are two modes of 3D display, automatic and eye control modes. In the automatic mode, the layered intersection of images is switched in accordance with the layer order for displays while the displayed intersection of image is switched by human eye in the eye control mode.

\section{IMPLEMENTATION AND EXPERIMENTAL RESULTS}

\section{Displayed Image in Automatic Mode}

Implementation of the proposed system is conducted. By using mouse operation by human eyes only, 3D image with different aspects can be recreated and display. It is confirmed that conventional image processing and analysis can be done with mouse operation by human eyes only.

Figure 2 shows the example of displayed layered images. In this example, 1024 of layer images are prepared for 3D object. By displaying the prepared layered images alternatively in automatic mode, 3D object appears on the screen. Furthermore, as shown in Figure 2, internal structure is visible other than the surface of the $3 \mathrm{D}$ object. It looks like a semi transparent 3D surface and internal structure in side of the $3 \mathrm{D}$ objects.
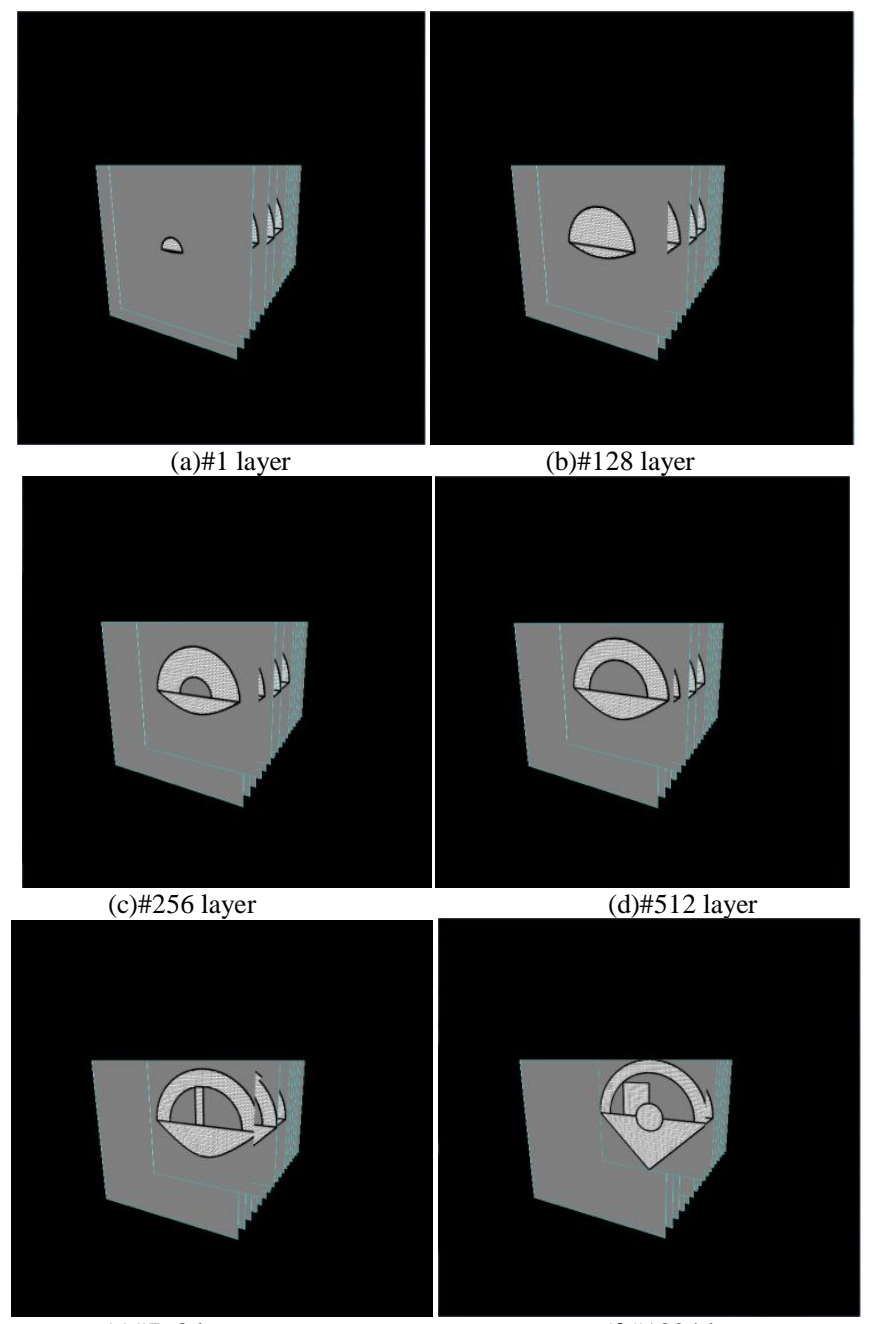

(e)\#768 laye

(f)\#1024 layer

Fig. 2. Example of displayed layered images.

\section{Displayed Image in Eye Cotroll Mode}

The configuration of the proposed system is shown in Figure 3. As shown in the figure, a user looking at the screen while camera put on top of screen display. 
Only camera which mounted on top of screen display is required. Optiplex 755 dell computer with Core 2 Quad 2.66 $\mathrm{GHz}$ CPU and $2 \mathrm{G}$ RAM is used. We develop our software under C++ Visual Studio 2005 and OpenCv Image processing Library which can be downloaded as free on their website. The specification of IR camera is shown in Table 1. The pointing value is determined based on head poses and eye gaze.

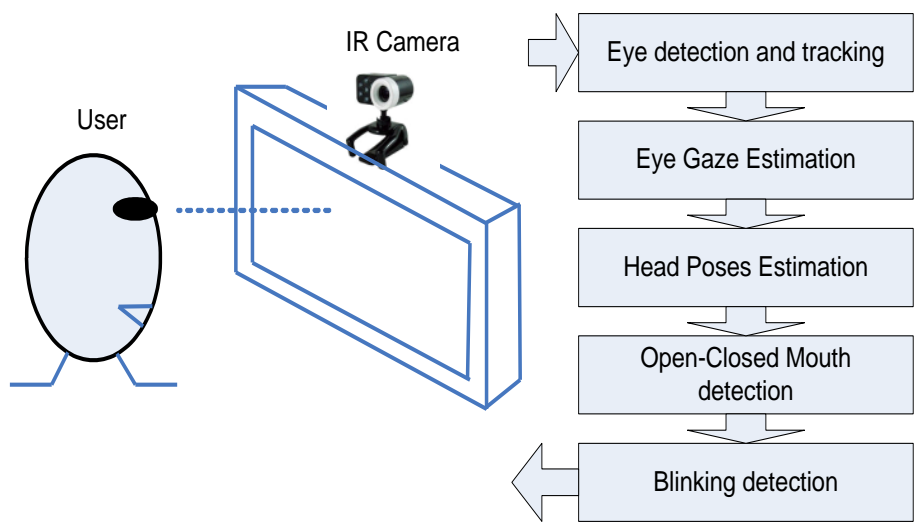

Fig. 3. Camera mouse configurations.

TABLE I. SPECIFICATION OF IR CAMERA

\begin{tabular}{|c|c|}
\hline Pixel & $1.3 \mathrm{M}$ \\
Resolution & $1280 \times 1024$ \\
Frame rate & $1280 \times 1024: 7.5 \mathrm{fps}, 640 \times 480: 30 \mathrm{fps}$ \\
Dimension & $52 \mathrm{~mm}(\mathrm{~W}) \times 65 \mathrm{~mm}(\mathrm{D}) \times 70 \mathrm{~mm}(\mathrm{H})$ \\
Weight & $85 \mathrm{~g}$ \\
Operating condition & $0-40 \mathrm{deg} . \mathrm{C}$ \\
Interface & USB 2.0 \\
IR Illumination & 7 IR LED \\
\hline
\end{tabular}

Figure 4 shows definition of success criteria of key input by human eye only.

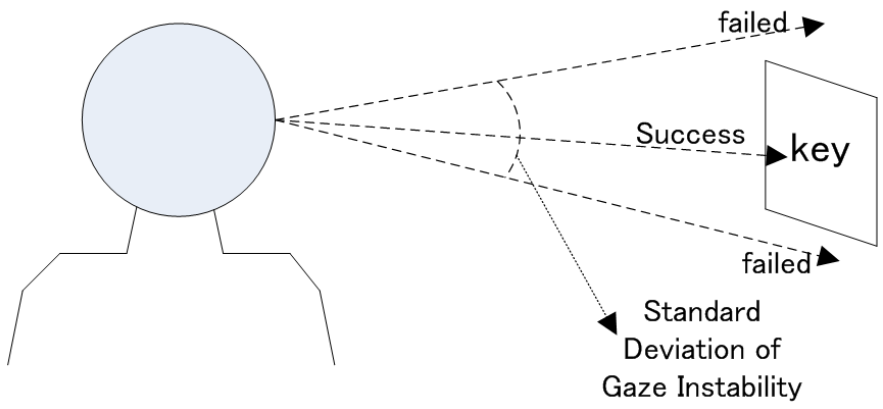

Fig. 4. Accuracy affected by gaze instability and key size

We classify the parameters affected by eye-based Human Computer Interaction: HCI in regard to accuracy as:

- $\quad$ instability of gaze,

- $\quad$ Size of keys and number of keys,

- Position of keys ("zero position" has maximum accuracy because it seems ideal for the eye).

Figure 4 shows how accuracy is affected by gaze instability and key size. The targeted key could be wrong hit if gaze instability is larger than key size. Gaze instability could be caused by:

- Poor camera image resolutions

- Noise,

- Environmental disturbances such as external light source.

As a result, the method for detecting eye performance has become somewhat unreliable. In addition to the aforementioned factors, distance between eye and target display have also played a part. Accuracy decreases with distance of subject from key and increases when subject gets closer. This phenomenon is a result of the flicker of the camera influencing the calculation of eye gaze, and accuracy is proportional to distance from the key.

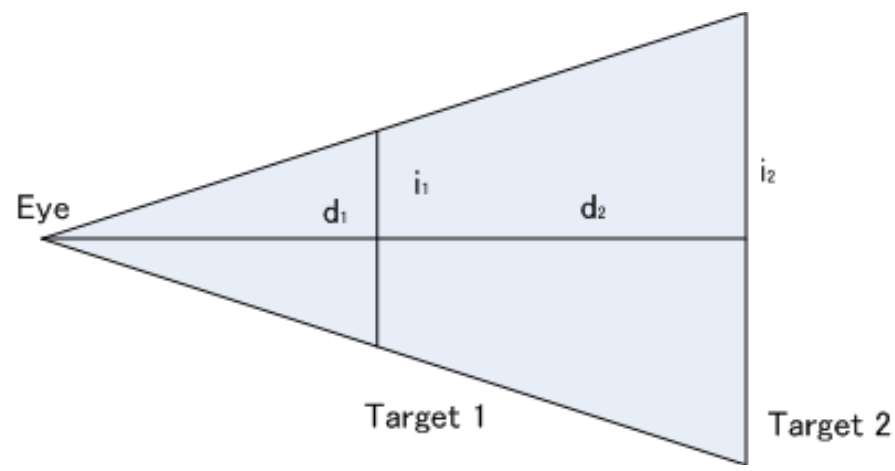

Fig. 5. Instability affected by distance of user-target

The relationship between instability and distance is depicted in Error! Reference source not found.5. It shows that the instability is proportional with distance formatted as follow,

$$
\frac{i_{1}}{i_{2}}=\frac{d_{1}}{d_{2}}
$$

where $i$ and $d$ are instability and distance respectively. Although gaze instability could be minimized, making it become perfect is difficult due to external disturbances. Now, we would like to show how we achieved perfect accuracy allowing for disturbances. If we assume that key size is $\mathrm{S}$ and the instability of gaze result is $\mathrm{SD}$, the accuracy can be formulated as,

$$
\begin{aligned}
& A \approx \frac{S}{S D} x 100 \%, \text { if SD } \geq \mathrm{S} \\
& A \approx 100 \%, \text { if } \mathrm{SD}<\mathrm{S}
\end{aligned}
$$

Gaze estimation accuracy is measured at the middle center to horizontally aligned five different locations with the different angles as shown in Figure 6. The experimental result shows the gaze estimation error at the center middle (No.1) shows zero while those for the angle ranges from -5 to 15 degrees is within 0.2 degree of angle estimation error as shown in Table 2. Because the user looks at the center middle with 5 degree allowance results in 0.2 degree of gaze estimation accuracy. 


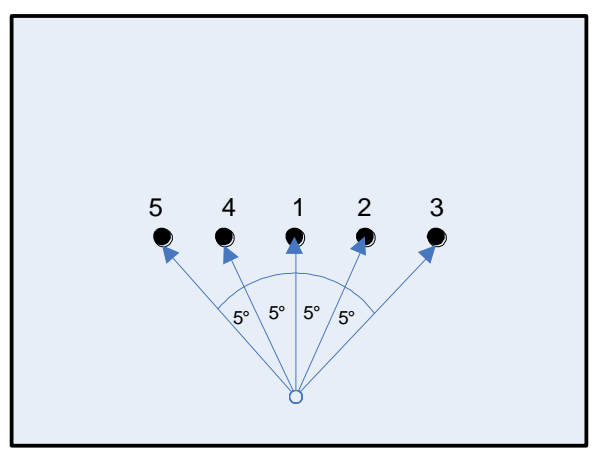

Fig. 6. Measuring accuracy

TABLE II. GAZE ESTIMATION ACCURACY AT THE DESIGNATED VIEWING ANGLES

\begin{tabular}{|c|c|c|c|c|c|}
\hline Point & $\mathbf{1}$ & $\mathbf{2}$ & $\mathbf{3}$ & $\mathbf{4}$ & $\mathbf{5}$ \\
\hline Error(degree) & 0 & 0.2 & 0.2 & 0.2 & 3.12 \\
\hline
\end{tabular}

In the eye control mode of 3D display, layer images are switched with human eyes only. Accidental blink is done within 0.3 seconds. Therefore, layer image is changed when user look at the corner of the image (the number of frame is written here) for more than 0.3 seconds (in this case, it is set at 0.7 seconds: 0.3 by $2+0.1$ second of margin). At the corner of the layer image, there is enlarged frame number as shown in Figure 7.

The frame number is displayed about one quarter of the screen size. Therefore, it does not require too much accuracy of the line of sight estimation. It is peripheral visual field; or peripheral vision for intersection of $3 \mathrm{D}$ object while the frame number is focused image.

Viewing at around the center of field of view allows acquisition of color and shape information of the objective 3D image while viewing of the peripheral visual fields allows movement and the location of 3D object pattern, in general. Therefore, throughout looking at frame number, user is looking at the 3D object in the peripheral visual fields and is looking at the frame number precisely in the eye control mode.

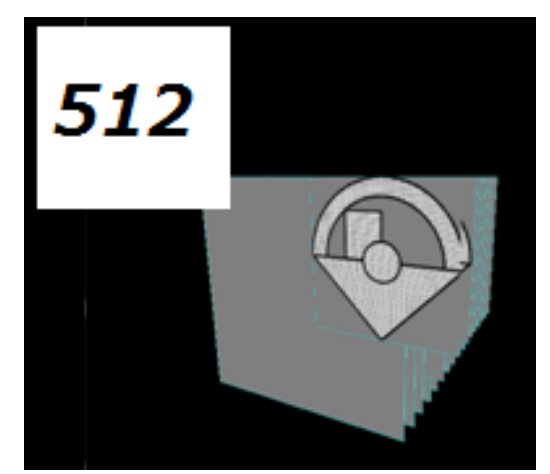

Fig. 7. User can select layer image by frame by frame by looking at the corner of the layer image
Thus layer selection for display can be done with human eye only. One of the applications of the proposed 3D object display is that LSI patter design as shown in Figure 8. It is getting easier to check through holes connectivity between layers by switching the displayed layer image by human eye only. Now a day, new model of LSI for mobile phone has to be delivered every three months. Therefore, there is a strong demand of LSI pattern design as much as they could. In this connection, the proposed eye control mode of layered image pattern representation is desired for this.

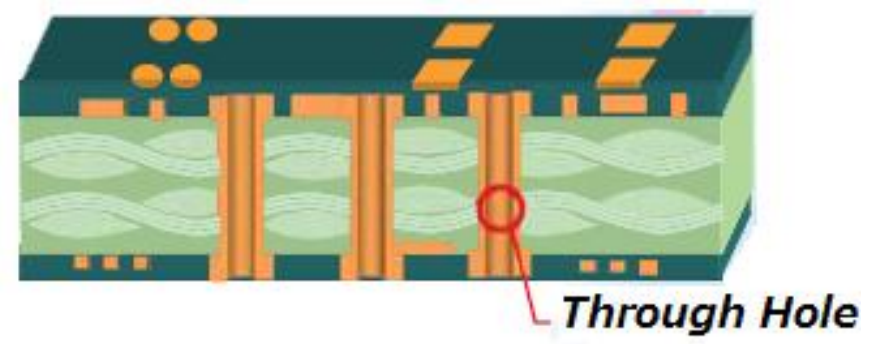

Fig. 8. Check the through holes of the layered LSI pattern with the proposed 3D object display method

E. Comparison of Processing Time Required for Switching Layer Images by betweenUusing Hands anf Fingers and Human Eye

Processing time by eye is as follows, Eye fixation $=230$ [it is ranged from 70 to 700 ] milliseconds Eye movement $=30$ milliseconds

Perceptual Processor $=100$ [it is ranged from 50 to 200] milliseconds

Cognitive Processor $=70$ [it is ranged from 25 to 170] milliseconds

On the other hand, the time required for press a key or button is as follows,

Best typist $=0.08$ seconds

Good typist $=0.12$ seconds

Average skilled typist $=0.20$ seconds

Average non-secretary $=0.28$ seconds

Typing random letters $=0.50$ seconds

Typing complex codes $=0.75$ seconds

Worst typist $=1.2$ seconds

These are measured by Card, Moran and Newell [16].

Meanwhile, the time required for point with a mouse (excluding click) $=1.1$ seconds. Furthermore, the time required for move hands to keyboard from mouse (or viceversa) $=0.4$ seconds in addition to mentally prepare $=1.35$ seconds.

Another experiments show that, in the eye control mode, it requires around 700 milliseconds for selection of function by human eye. On the other hand, it requires 1.5 to 2 seconds for selection of function by human hand and fingers.

As a conclusion, the time required for mouse operations and or key-in operations by human eye is faster than that by hand and or fingers. Therefore, eye control mode of operations is faster than hand / finger operations. In particular for LSI pattern design of which it requires quick operation, eye control 
mode is useful because through hole between LSI layers can be checked easily and fast.

\section{CONCLUSION}

Method for 3D rendering based on intersection image display which allows representation of internal structure is proposed. The proposed method is essentially different from the conventional volume rendering based on solid model which allows representation of just surface of the 3D objects. By using afterimage, internal structure can be displayed through exchanging the intersection images with internal structure for the proposed method.

Through experiments with CT scan images, the proposed method is validated. Also one of other applicable areas of the proposed for design of 3D pattern of Large Scale Integrated Circuit: LSI is introduced. Layered patterns of LSI can be displayed and switched by using human eyes only. It is confirmed that the time required for displaying layer pattern and switching the pattern to the other layer by using human eyes only is much faster than that using hands and fingers. It is also found the followings,

Afterimage based 3D object representation onto 2D display works well

Automatic and manual (eye control) modes are available

Automatic mode works for continuous display while manual mode works for detail display in accordance with users' intension.

\section{ACKNOWLEDGMENT}

The author would like to thank Mr. Guo XiaoYu of Saga University and Arai's laboratory members for their valuable discussions and suggestions through this research works.

\section{REFERENCES}

[1] K.Arai, H.Uwataki, Computer input by human eyes only based on cornea center extraction which allows users' movements, Journal of Institute of Electric Engineering of Japan, C-127, 7, 1107-1114, 2007.

[2] K.Arai, M.Yamaura, Improvement of blink detection performance based on Morphologic filter for computer input by human eyes only, Journal of Image Electronics Engineering Society of Japan, 37, 5, 601-609, 2008.

[3] K.Arai, K.Yajima, Communication aid based on computer input by human eyes only, Journal of Institute of Electric Engineering of Japan, C-128, 11, 1679-1686, 2008

[4] D. Purwanto, R. Mardiyanto and K. Arai, Electric wheel chair control with gaze detection and eye blinking, Artificial Life and Robotics, AROB Journal, 14, 694,397-400, 2009.
[5] K.Arai, R. Mardiyanto, Computer input by human eyes only with blink detection based on Gabor filter, Journal of Visualization Society of Japan, 29,Suppl.2, 87-90,2009.

[6] K. Arai and R. Mardiyanto, Real time blinking detection based on Gabor filter, International Journal of Human Computer Interaction, 1, 3, 33-45, 2010.

[7] K. Arai and M. Yamaura, Computer input with human eyes only using two Purkinje images which works in a real time basis without calibration, International Journal of Human Computer Interaction, 1, 3, 71-82, 2010.

[8] [8] K. Arai and R. Mardiyanto, Camera mouse and keyboard for handicap person with trouble shooting capability, recovery and complete mouse events, International Journal of Human Computer Interaction, 1, 3, 46-56, 2010.

[9] K. Arai, R. Mardiyanto, A prototype of electric wheel chair control by eye only for paralyzed user, Journal of Robotics and Mechatronics, 23, 1, 66-75, 2010.

[10] K. Arai, K. Yajima, Robot arm utilized having meal support system based on computer input by human eyes only, International Journal of Human-Computer Interaction, 2, 1, 120-128, 2011.

[11] Kohei Arai, Ronny Mardiyanto, Autonomous control of eye based electric wheel chair with obstacle avoidance and shortest path finding based on Dijkstra algorithm, International Journal of Advanced Computer Science and Applications, 2, 12, 19-25, 2011

[12] K. Arai, R. Mardiyanto, Eye-based human-computer interaction allowing phoning, reading e-book/e-comic/e-learning, Internet browsing and TV information extraction, International Journal of Advanced Computer Science and Applications, 2, 12, 26-32, 2011.

[13] K. Arai, R. Mardiyanto, Eye based electric wheel chair control systemI(eye) can control EWC-, International Journal of Advanced Computer Science and Applications, 2, 12, 98-105, 2011.

[14] K. Arai, R. Mardiyanto, Evaluation of users' impact for using the proposed eye based HCI with moving and fixed keyboard by using eeg signals, International Journal of Research and Reviews on Computer Science, 2, 6, 1228-1234, 2011.

[15] K. Arai, R. Mardiyanto, Electric wheel chair controlled by human eyes only with obstacle avoidance, International Journal of Research and Reviews on Computer Science, 2, 6, 1235-1242, 2011.

[16] Card, Stuart; Thomas P. Moran and Allen Newell (1983). The Psychology of Human Computer Interaction. Lawrence Erlbaum Associates. ISBN 0-89859-859-1.

\section{AUTHORS PROFILE}

Kohei Aarai He received BS, MS and PhD degrees in 1972, 1974 and 1982, respectively. He was with The Institute for Industrial Science and Technology of the University of Tokyo from April 1974 to December 1978 and also was with National Space Development Agency of Japan from January, 1979 to March, 1990. During from 1985 to 1987, he was with Canada Centre for Remote Sensing as a Post Doctoral Fellow of National Science and Engineering Research Council of Canada. He moved to Saga University as a Professor in Department of Information Science on April 1990. He was a councilor for the Aeronautics and Space related to the Technology Committee of the Ministry of Science and Technology during from 1998 to 2000. He was a councilor of Saga University for 2002 and 2003. $\mathrm{He}$ also was an executive councilor for the Remote Sensing Society of Japan for 2003 to 2005. He is an Adjunct Professor of University of Arizona, USA since 1998. He also is Vice Chairman of the Commission-A of ICSU/COSPAR since 2008. He wrote 30 books and published 332 journal papers. 